\title{
Double - Diffusive Convection of Non - Newtonian Walters' (MODELB') Viscoelastic Fluid Through Brinkman Porous Medium With Suspended Particles
}

\author{
K. Thirumurugan ${ }^{* 1}$ and R. Vasanthakumari ${ }^{2}$ \\ ${ }^{* 1}$ Research Scholar, Research and Development Centre, Bharathiar University, Coimbatore, India \\ ${ }^{2}$ Associate Professor, Kanchima Munivar Centre for PG Studies, Puducherry, India
}

Email: thirumurugan.kirithish@gmail.com

\begin{abstract}
In this paper, the double -diffusive convection of Non - Newtonian Walters'B' viscoelastic fluid with suspended particles through Brinkman porous medium is considered. Following the linearized stability theory and normal mode analysis, the dispersion relation is obtained. For stationary convection, it is found that the Darcy number and stable solute gradient have stabilizing effects whereas the suspended particles and medium permeability have destabilizing effects on the system. Graphs have been represented by giving numerical values to the parameters to depict the stability characteristics. The stable solute gradient and viscoelasticity, suspended particles, gravity field and medium permeability are found to introduce oscillatory modes in the system which is non - existent in their absence.
\end{abstract}

Keywords: Walters'B' fluid, Double-Diffusive convection, Compressibility, Brinkman porous medium, Viscoelasticity.

\section{INTRODUCTION}

Chandrasekhar [1] has taken a detailed research in theoretical and experimental results of the onset of thermal instability (Bénard Convection) in a fluid layer under varying assumptions of hydrodynamics and hydromagnetics stability for viscoelastic fluids. Veronis [2] has considered the problem of thermohaline convection in a layer of fluid heated from below and subjected to a stable salinity gradient. Stommel and Fedorov [3] have developed that the length scales characteristic of double-diffusive convecting layers in the oceans could be sufficiently large for Earth's rotation to become important in their formation. Moreover, the rotation of the Earth distorts the boundaries of a hexagonal convection cell in a fluid through a porous medium and the distortion plays an important role in the extraction of energy in the geothermal regions. Brakke [5] has dealt with a double-diffusive instability that occurs when a solution of a slowly diffusing protein is laid over a denser solution of more rapidly diffusing sucrose. Nason et al. [6] have studied that the thermal instability is deleterious to certain biochemical separations may be suppressed by rotation in the ultracentrifuge. Chandra [13] has explained a contrary between the theory and experiment for the onset of convection in fluids heated from below.

In geophysical situations the fluid is often not pure but contains suspended particles. Scanlon and Segel [7] have considered the effects of suspended particles under onset of
Bénard convection and found that the critical Rayleigh number is reduced solely because of the heat capacity of the pure gas was supplemented by the particles. Here, the suspended particles where thus found to destabilize the layer. Palaniswamy and Purushotham [8] have studied the stability of shear flow of stratified fluids with fine dust and found the effects of fine dust to increase the region of instability.

The Boussinesq approximation has been derived throughout the system which explicits that variations of density in the equations of motion can safely be neglected everywhere except in its association with the external force. For incompressible fluids the approximation is well justified, but in the case of compressible fluids forming the governing equations of the system become quiet complicated.On simplifying them Boussinesq tried to justify the approximation for compressible fluids when the density variations arise principally from the thermal effects. Spiegel and Veronis [9] have simplified the set of compressible fluid flow governing equations under the following assumptions:

(a) The depth of the fluid layer is much less than the scale - height, as defined by them, and

(b) The fluctuations in temperatures, density and pressure, introduced due to motion, do not exceed the total static variations.

Sharma and Rana [4] have also expounded the thermosolutal instability of incompressible walters' (MODEL $\left.B^{\prime}\right)$ rotating fluid permeated with suspended particles and variable gravity field in a porous medium. Bhatia and Steiner 
[10] have to account for the problem of thermal instability of Maxwellian viscoelastic fluid in the presence of rotation and have found the rotation has a destabilizing influence in contrast to the stabilizing effect on a ordinary viscous (Newtonian) fluid. Sharma [11] has posited the thermal instability of an oldroydian viscoelastic fluid acted on a uniform rotation. The convective flow in a porous medium using linear stability theory has been examined by Lapwood [14]. Wooding [15] has considered the Rayleigh instability of a thermal boundary layer inflow through a porous medium. Parul Aggarwal and Urvasi Gupta [16] have discussed double-diffusive convection of compressible rotating Walters'B' fluid with Hall current saturating a porous medium. Thirumurugan and Vasanthakumari [18] studied the stability of hydromagnetics Walters' $\mathrm{B}^{\prime}$ viscoelastic fluid rotating in porous medium.

The influence of non - Newtonian fluids on geophysics, chemical industry and petroleum goelogy is gaining momentum. Walters' [12] has reported the mixture of polymethyl methacrylate and pyridine heated at $25^{\circ} \mathrm{C}$ containing with $30.5 \mathrm{~g}$ of polymer per litre behaves very nearly as the Walters' (MODELB') elastic-viscous fluid. Such and other polymer are used in agriculture, communication applications and in biomedical applications. The Walters'B' elastic - viscous fluid is one such fluid. Thirumurugan and Vasanthakumari [17] have studied the thermal convection in walters'B' viscoelastic fluid in DarcyBrinkman porous medium with effect of dusty particles.

The paramountcy of geophysical fluid dynamics, biophysical field and various applications mentioned above, the double-diffusive convection Non-Newtonian Walters' (MODELB') viscoelastic fluid with suspended particles through Brinkman porous medium have been enforced to consider in the present paper.

\section{MATHEMATICAL MODEL AND PERTURBATION EQUATIONS}

Consider a horizontal and an infinite compressible Walters' $\mathrm{B}^{\prime}$ viscoelastic fluid layer of depth $d$, in an isotropic and homogeneous medium of porosity $\varepsilon$ and permeability $k_{1}$ which is bounded the by planes $z=0$ and $z=d$ and that a uniform temperature gradient $\beta=(|d T / d z|)$, and a uniform solute gradient $\beta^{\prime}=(|d C / d z|)$ are maintained. The gravity field $\mathbf{g}(0,0,-g)$, and a uniform vertical magnetic field $\mathbf{H}(0,0$, $H)$, act on the system.

The governing equations of motion, continuity, heat conduction, and Maxwell's equation for the flow of Walters'B' fluids are,

$$
\begin{gathered}
\frac{\rho}{\varepsilon}\left[\frac{\partial v}{\partial t}+\frac{1}{\varepsilon}(\boldsymbol{v} \cdot \nabla) \boldsymbol{v}\right]=-\nabla p+\boldsymbol{g} \rho-\frac{1}{k_{1}}\left(\mu-\mu^{\prime} \frac{\partial}{\partial t}\right) \boldsymbol{v}+ \\
\tilde{\mu} \nabla^{2} \boldsymbol{v}+\frac{\boldsymbol{K}^{\prime} \boldsymbol{N}}{\boldsymbol{\varepsilon}}\left(\boldsymbol{v}_{\boldsymbol{d}}-\boldsymbol{v}\right)
\end{gathered}
$$

$\frac{\partial q}{\partial t}+\nabla(\rho . v)=0$

$\rho C_{f}\left(\frac{\partial}{\partial t}+\mathbf{v} \cdot \nabla\right) T+m N C_{p t}\left[\varepsilon \frac{\partial}{\partial t}+v_{d} \cdot \nabla\right] T=K_{T} \nabla^{2} T$

$\rho C_{f}^{\prime}\left(\frac{\partial}{\partial t}+\mathbf{v} \cdot \nabla\right) C+m N C_{p t}{ }^{\prime}\left[\varepsilon \frac{\partial}{\partial t}+v_{d} \cdot \nabla\right] C=K_{T} \nabla^{2} C$

where $v_{d}(\bar{x}, t)$ and $N(\bar{x}, t)$ are indicating the velocity, number density of the particles respectively, $K^{\prime}=6 \pi \eta \rho v$ is the strokes drag coefficient, here $\eta$ is the particle radius, $v_{d}=(l, r, s)$ and $\bar{x}=(x, y, z)$.

The equations of motion and continuity for the particles are

$m N\left[\frac{\partial v_{d}}{\partial t}+\frac{1}{\varepsilon}\left(\boldsymbol{v}_{\boldsymbol{d}} \cdot \boldsymbol{\nabla}\right) \boldsymbol{v}_{\boldsymbol{d}}\right]=\mathrm{K}^{\prime} \mathrm{N}\left(\boldsymbol{v}-\boldsymbol{v}_{\boldsymbol{d}}\right)$

where $m N$ is the mass of the particles per unit volume.

$\varepsilon \frac{\partial N}{\partial T}+\nabla \cdot\left(N v_{d}\right)=0$

The presence of particles adds an extra force term proportional to the velocity difference between particles and fluid and appears in the equation of motion. Since the force exerted by the fluid on the particles is equal and opposite to that exerted by the particles on the fluid, there must be an extra force term, equal in magnitude but opposite sign, in the equation of the motion for the particles. The buoyancy force on the particle is neglected. Interparticles reactions are not considered either since the distance between the particles is quite large compared with their diameters. These assumptions have been used in writing the equation of motion for the particles.

Spiegel and Veronis [9] have expressed any state variables pressure, density and temperature are expressed in the form

$f(x, y, z, t)=f_{m}+f_{0}(z)+f^{\prime}(x, y, z, t)$,

where $f_{m}$ denotes for constant space distribution $f, f_{0}$ is the variation in the absence of motion, and $(x, y, z, t)$ is the fluctuations resulting from motion. The initial state of the system is

$$
\begin{aligned}
& p=p(z), \rho=\rho(z), T=T(z), v=(0,0,0), C=C(z), \\
& v=(0,0,0), N=N_{0}
\end{aligned}
$$

where

$$
\begin{aligned}
& p(z)=p_{m}-g \int_{0}^{z}\left(\rho_{m}+\rho_{0}\right) d z, \rho(z) \\
& =\rho_{m}\left[1-\alpha_{m}\left(T-T_{0}\right)+\alpha^{\prime}\left(C-C_{0}\right)+K_{m}\left(p-p_{m}\right)\right], \\
& T=-\beta z+T_{0}, C=-\beta^{\prime} z+C_{0}, \alpha_{m}=-\left(\frac{1}{\rho} \frac{\partial \rho}{\partial C}\right)_{m}, \\
& K_{m}=\left(\frac{1}{\rho} \frac{\partial \rho}{\partial p}\right)_{m}
\end{aligned}
$$

Here, $p_{m}$ and $\rho_{m}$ denote a constant space distribution of $p$ while $T_{0}$ and $\rho_{0}$ denote temperature and density of the fluid at the lower boundary.

Spiegel and Veronis [9] has given results for the compressible fluid and the flow equations are found to be the same as that of incompressible fluid except that the static temperature gradient $\beta$ is replaced by the excess over the adiabatic $\left(\beta-\frac{g}{C_{p}}\right), C_{p}$ being specific heat of the fluid at the constant pressure. This is an exact solution to the governing equations.

Then, the change in density $\delta \rho$ caused by the perturbation $\theta$ in temperature is given by

$\delta \rho=-\rho_{m}\left(\alpha \theta-\alpha^{\prime} \gamma\right)$ 
The linearized perturbation equations governing the fluid are

$\frac{1}{\varepsilon} \frac{\partial \boldsymbol{v}}{\partial t}=-\frac{1}{\rho_{m}}(\nabla \delta p)-\boldsymbol{g}\left(\alpha \theta-\alpha^{\prime} \gamma\right)-\frac{1}{k_{1}}\left(v-v^{\prime} \frac{\partial}{\partial t}\right) \boldsymbol{v}$

$+\frac{\overline{\boldsymbol{\mu}}}{\boldsymbol{\rho}_{m}} \nabla^{2} \boldsymbol{v}+\frac{K^{\prime} N_{0}}{\rho_{0} \varepsilon}\left(\boldsymbol{v}_{\boldsymbol{d}}-\boldsymbol{v}\right)$

$\nabla \cdot v=0$

$(1+b \varepsilon) \frac{\partial \theta}{\partial t}=\left(\beta-\frac{g}{C_{p}}\right)(w+b s)+K \nabla^{2} \theta$

$\left(1+b^{\prime} \varepsilon\right) \frac{\partial \theta}{\partial t}=\left(\beta-\frac{g}{C_{p}}\right)\left(w+b^{\prime} s\right)+K^{\prime} \nabla^{2} \gamma$

$m N_{0} \frac{\partial v_{d}}{\partial t}=K^{\prime} N_{0}\left(\boldsymbol{v}-\boldsymbol{v}_{\boldsymbol{d}}\right)$

$\frac{\partial M_{d}}{\partial t}+\nabla \cdot \boldsymbol{v}_{\boldsymbol{d}}=\mathbf{0}$

where $v=\frac{\mu}{\rho_{m}}, v^{\prime}=\frac{\mu^{\prime}}{\rho_{m}}, K=\frac{K_{T}}{\rho_{m} C_{f}}, \frac{g}{c_{f}}$ and $w$ denote the kinematic viscosity, kinematic viscoelasticity, thermal diffusivity, solute diffusivity, adiabatic gradient and vertical fluid velocity, respectively.

Also, $b=\frac{m N c_{p} t}{\rho_{0} c_{f}}, b^{\prime}=\frac{m N C_{p t} t^{\prime}}{\rho_{0} c_{f}^{\prime}}$ and $w, s$ are the vertical fluid and particles velocity.

In Cartesian form, Eqs. (11) - (13) with the help of Eqs. (15) and (16) can be expressed as

$$
\begin{gathered}
\frac{1}{\varepsilon} \frac{\partial \boldsymbol{u}}{\partial t}=-\frac{1}{\rho_{m}} \frac{\partial}{\partial x}(\delta p)-\frac{1}{k_{1}}\left(v-v^{\prime} \frac{\partial}{\partial t}\right) u+\frac{\overline{\boldsymbol{\mu}}}{\boldsymbol{\rho}_{\mathbf{0}}} \nabla^{2} u \\
-\frac{m N_{0}}{\in\left(\frac{m \partial}{\mathrm{K}^{\prime} \partial t}+1\right) \boldsymbol{\rho}_{m}} \frac{\partial \boldsymbol{u}}{\partial t},
\end{gathered}
$$

$$
\begin{aligned}
\frac{1}{\varepsilon} \frac{\partial \boldsymbol{v}}{\partial t}= & -\frac{\mathbf{1}}{\boldsymbol{\rho}_{\mathbf{0}}} \frac{\partial}{\partial y}(\delta p)-\frac{1}{k_{1}}\left(v-v^{\prime} \frac{\partial}{\partial t}\right) \boldsymbol{v}+\frac{\overline{\boldsymbol{\mu}}}{\boldsymbol{\rho}_{\mathbf{0}}} \nabla^{2} v \\
& -\frac{m N_{0}}{\in\left(\frac{m \partial}{\mathrm{K}^{\prime} \partial t}+1\right) \boldsymbol{\rho}_{\mathbf{0}}} \frac{\partial v}{\partial t^{\prime}}
\end{aligned}
$$

$\begin{aligned} \frac{1}{\varepsilon} \frac{\partial \boldsymbol{w}}{\partial t}= & -\frac{\mathbf{1}}{\boldsymbol{\rho}_{\mathbf{0}}} \frac{\partial}{\partial z}(\delta p)+g \alpha \theta-\frac{1}{k_{1}}\left(v-v^{\prime} \frac{\partial}{\partial t}\right) \boldsymbol{w}+\frac{\overline{\boldsymbol{\mu}}}{\boldsymbol{\rho}_{\boldsymbol{m}}} \nabla^{2} \boldsymbol{w} \\ & -\frac{m N_{0}}{\epsilon\left(\frac{m \partial}{\mathrm{K}^{\prime} \partial t}+1\right) \boldsymbol{\rho}_{\boldsymbol{m}}} \frac{\partial \boldsymbol{w}}{\partial t}\end{aligned}$

$\frac{\partial u}{\partial x}+\frac{\partial v}{\partial y}+\frac{\partial w}{\partial z}=0$

$(1+b \varepsilon) \frac{\partial \theta}{\partial t}=\left(\beta-\frac{g}{c_{p}}\right)(w+b s)+K \nabla^{2} \theta$

$\left(1+b^{\prime} \varepsilon\right) \frac{\partial \theta}{\partial t}=\beta^{\prime}\left(w+b^{\prime} s\right)+K^{\prime} \nabla^{2} \gamma$

Operating Eqs. (17) and (18) by $\frac{\partial}{\partial x}$ and $\frac{\partial}{\partial y}$ respectively, adding, we get

$$
\begin{aligned}
\frac{1}{\varepsilon} \frac{\partial}{\partial t}\left(\frac{\partial \boldsymbol{w}}{\partial z}\right)= & -\frac{\mathbf{1}}{\boldsymbol{\rho}_{\boldsymbol{m}}} \frac{\partial}{\partial x}\left(\nabla^{2}-\frac{\partial^{2}}{\partial z^{2}}\right)-\frac{1}{k_{1}}\left(v-v^{\prime} \frac{\partial}{\partial t}\right)\left(\frac{\partial \boldsymbol{w}}{\partial z}\right) \\
& +\frac{\overline{\boldsymbol{\mu}}}{\boldsymbol{\rho}_{\mathbf{0}}} \nabla^{2}\left(\frac{\partial \boldsymbol{w}}{\partial z}\right)-\frac{m N_{0}}{\epsilon\left(\frac{m \partial}{\mathrm{K}^{\prime} \partial t}+1\right) \boldsymbol{\rho}_{\boldsymbol{m}}} \frac{\partial}{\partial t}\left(\frac{\partial \boldsymbol{w}}{\partial z}\right)
\end{aligned}
$$

Operating Eqs. (19) and (22) by $\left(\nabla^{2}-\frac{\partial^{2}}{\partial z^{2}}\right)$ and $\frac{\partial}{\partial z}$ respectively and adding to eliminate $\delta p$ between Eqs. (19) and (22), we get

$$
\begin{aligned}
\frac{1}{\varepsilon} \frac{\partial}{\partial t}\left(\nabla^{2} w\right)= & -\frac{1}{k_{1}}\left(v-v^{\prime} \frac{\partial}{\partial t}\right)\left(\nabla^{2} w\right)+\frac{\overline{\boldsymbol{\mu}}}{\boldsymbol{\rho}_{\mathbf{0}}}\left(\nabla^{4} w\right) \\
& +\left(\frac{\partial^{2}}{\partial x^{2}}+\frac{\partial^{2}}{\partial y^{2}}\right) \alpha \theta-\frac{m N_{0}}{\epsilon\left(\frac{m \partial}{\mathrm{K}^{\prime} \partial t}+1\right) \boldsymbol{\rho}_{\boldsymbol{m}}} \frac{\partial}{\partial t}\left(\nabla^{2} \mathrm{w}\right)
\end{aligned}
$$

where $\nabla^{2}=\frac{\partial^{2}}{\partial x^{2}}+\frac{\partial^{2}}{\partial y^{2}}+\frac{\partial^{2}}{\partial z^{2}}$.

\section{THE DISPERSION RELATION}

To decompose the perturbations into normal modes by seeking solutions in the form of functions $(x, y, t)$,

$[w, \theta]=[W(z), S(z), \Theta(z), \Gamma(z)] \exp \left(i k_{x}^{x}+i k_{y}^{y}+n t\right)$

where $k_{x}$ and $k_{y}$ are the wave numbers in $x$ and $y$ directions $k=\sqrt{k_{x}^{2}+k_{y}^{2}}$ is the resultant wave numbers, and $n$ is in general, a complex constant.

Using Eq. (25) in Eqs. (23), (21) and (22) become

$$
\begin{aligned}
\frac{n}{\varepsilon}[ & \left.\frac{d^{2}}{d z^{2}}-k^{2}\right] W \\
= & -g k^{2}\left(\alpha \theta-\alpha^{\prime} \gamma\right)-\frac{1}{k_{1}}\left(v-v^{\prime} n\right)\left(\frac{d^{2}}{d z^{2}}-k^{2}\right) W \\
& +\frac{\bar{\mu}}{\rho_{0}}\left(\frac{d^{2}}{d z^{2}}-k^{2}\right) W-\frac{m N_{0}}{\epsilon\left(\frac{m \partial}{K^{\prime} \partial t}+1\right) \rho_{m}}\left(\frac{d^{2}}{d z^{2}}-k^{2}\right) W \\
& (1+b \varepsilon) \frac{\partial \theta}{\partial t}=\left(\beta-\frac{g}{c_{p}}\right)(w+b s)+K\left(\frac{d^{2}}{d z^{2}}-k^{2}\right) \Theta \\
& \left(1+b^{\prime} \varepsilon\right) \frac{\partial \theta}{\partial t}=\beta^{\prime}\left(w+b^{\prime} s\right)+K^{\prime} \nabla^{2} \gamma
\end{aligned}
$$

Eqs. (26) and (28) in non - dimensional form, become

$$
\begin{aligned}
& {\left[1+\left(\frac{p_{l}}{\varepsilon}+\frac{M p_{l}}{\varepsilon\left(1+\tau_{1} \sigma\right)}+F\right) \sigma-D_{A}\left(D^{2}-a^{2}\right)\right]\left(D^{2}-a^{2}\right) W} \\
& +\frac{g a^{2} d^{2} p_{l} \alpha \Theta}{v}-\frac{g p_{l a^{2} d^{2} \alpha^{\top} \mathrm{T}}}{v}=0 \\
& {\left[D^{2}-a^{2}-E_{1} P_{1} \sigma\right] \Theta=-\beta \frac{d^{2}}{K}\left(\frac{G-1}{G}\right)\left(\frac{B+\tau_{1} \sigma}{1+\tau_{1} \sigma}\right) W} \\
& {\left[D^{2}-a^{2}-E_{1}^{\prime} P_{1}^{\prime} \sigma\right] \Gamma=\left(-\beta^{\prime d^{2}} K^{\prime}\right)\left(\frac{B^{\prime}+\tau_{1} \sigma}{1+\tau_{1} \sigma}\right) W}
\end{aligned}
$$

where we have put

$$
E_{1}=1+b \varepsilon, E_{1}^{\prime}=1+b^{\prime} \varepsilon^{\prime}, B=b+1, B^{\prime}=1+b^{\prime}, a=
$$
$k d, \sigma=\frac{n d^{2}}{v}, P_{1}=\frac{V}{\chi}, F=\frac{v^{\prime}}{d^{2}}$ and $p_{l}=\frac{k_{1}}{d^{2}}, \quad$ is the dimensionless medium permeability, $p_{1}=\frac{v}{k}$, is the themal Prandtl number and $D_{A}=\frac{\widetilde{\widetilde{\mu}} k_{1}}{\mu d^{2}}$, is the Darcy number modified by the viscosity ratio.

Eliminating $\Theta$ and $\Gamma$ between Eqs. (29) and (31), we obtain 
$\left[1+\left(\frac{p_{l}}{\varepsilon}+\frac{M p_{l}}{\varepsilon\left(1+\tau_{1} \sigma\right)}+F\right) \sigma-D_{A}\left(D^{2}-a^{2}\right)\right]\left(D^{2}-a^{2}\right)$

$\left(D^{2}-a^{2}-E_{1} P_{1} \sigma\right)\left(D^{2}-a^{2}-E_{1}^{{ }^{\prime} P_{1}{ }^{\prime} \sigma}\right) W$

$+S a^{2}\left(\frac{B^{\prime}+\tau_{1} \sigma}{1+\tau_{1} \sigma}\right)\left(D^{2}-a^{2}-E_{1} P_{1} \sigma\right) W$

$-R a^{2} P_{1}\left(\frac{G-1}{G}\right)\left(\frac{B+\tau_{1} \sigma}{1+\tau_{1} \sigma}\right)\left(D^{2}-a^{2}-E_{1} P_{1} \sigma\right) W=0$

where $R=\left(\frac{g \alpha \beta d^{4}}{v k}\right)$ is the thermal Rayleigh number and $S=$ $\left(\frac{g \alpha^{\prime} \beta^{\prime} d^{4}}{v k^{\prime}}\right)$ is the analogous solute Rayleigh number.

Assume that the temperature at the boundaries is kept fixed, the fluid layer is confirmed between two boundaries and adjoining medium is electrically non - conducting. The boundary conditions appropriate to the problem are $W=D^{2} W=0, \quad D Z=0, \Theta=0 \quad$ at $z=0, z=1$,

The case of two boundaries, though a little artificial is the most appropriate for stellar atmospheres. Using the boundary conditions, all the even order derivatives of $W$ characterizing the lowest mode is

$W=W_{0} \sin \pi z$,

where $W_{0}$ is a constant.

Substituting Eq. (27) in Eq. (25) we get

$R_{1} x P=\left(\frac{G}{G-1}\right)\left[1+\left(\frac{p_{l}}{\varepsilon}+\frac{M P}{\varepsilon\left(1+\tau_{1} i \sigma_{1}\right)}+\pi^{2} F\right) i \sigma_{1}+\right.$

$\left.D_{A 1}(1+x)\right](1+x)\left(1+x+E_{1} p_{1} i \sigma_{1}\right)+\left(\frac{1+i \tau_{1} \pi^{2} i \sigma_{1}}{B+i \tau_{1} \pi^{2} i \sigma_{1}}\right)+$

$\frac{S_{1} P x\left(1+x+E_{1} p_{1} i \sigma_{1}\right)}{\left(1+x+E_{1}^{\prime} p_{1}^{\prime} \sigma\right.}\left(\frac{B^{\prime}+i \tau_{1} \pi^{2} i \sigma_{1}}{B+\tau_{1} \pi^{2} i \sigma_{1}}\right)$

where

$\mathrm{R}_{1}=\frac{\mathrm{R}}{\pi^{4}}, \mathrm{~S}_{1}=\frac{\mathrm{s}}{\pi^{4}} \mathrm{~T}_{\mathrm{A} 1}=\frac{\mathrm{T}_{\mathrm{A}}}{\pi^{4}}, \mathrm{D}_{\mathrm{A} 1}=\frac{\mathrm{D}_{\mathrm{A}}}{\pi^{2}}, \mathrm{x}=\frac{\mathrm{a}^{2}}{\pi^{2}}, \mathrm{i} \sigma_{1}=$ $\frac{\sigma}{\pi^{2}}, P=\pi^{2} p_{1}, \tau=\frac{m}{K^{\prime}}, \tau_{1}=\frac{\tau v}{d^{2}}, M=\frac{m_{0}}{\rho_{m}}$.

Eq. (35) is required dispersion relation accounting for the onset of double-diffusive convection of Walters' $B^{\prime}$ elastico - viscous fluid permeated with suspended particles in a Brinkman porous medium.

\section{THE STATIONARY CONVECTION}

For stationary convection putting $\sigma=0$ in Eq. (35), we obtain

$R_{1}=\left(\frac{G}{G-1}\right)\left\{\frac{(1+x)^{2}}{x P B}\left[1+(1+x) D_{A 1}\right]+\frac{S_{1} B^{\prime}}{B}\right\}$

Eq. (36) expresses the modified Rayleigh number $R_{1}$ as a function of the dimensionless wave number $\mathrm{x}$ and the parameters $G, B, D_{A 1}, P$ and Walters'B' elastic - viscous fluid behaves like an ordinary Newtonian fluid since elastico viscous parameter $F$ vanishes with $\sigma$. Let the non dimensional numbers $G$ accounting for compressibility effect is kept as fixed, then we get
$\overline{R_{C}}=\left(\frac{G}{G-1}\right) R_{C}$

$\overline{R_{C}}$ and $R_{C}$ denotes, respectively, the critical number in the presence and absence of compressibility thus, the effect of compressibility is to postpone the instability on the onset of double diffusive convection. The cases $G=1$ and $G<1$ correspond to infinite and negative values of Rayleigh numbers due to compressibility which are not relevant to the present study.

To study the effect of Darcy number, stable solute gradient, suspended particles and medium permeability, we examine the behavior of $\frac{d R_{1}}{d D_{A 1}}, \frac{d R_{1}}{d S_{1}}, \frac{d R_{1}}{d B}$, and $\frac{d R_{1}}{d P}$ analytically.

From Eq. (36), we get

$\frac{d R_{1}}{d D_{A 1}}=\left(\frac{G}{G-1}\right) \frac{(1+x)^{3}}{x P B}$

which is positive implying thereby the stabilizing effect of Darcy number on the double diffusive convection in Walters'B' elastico-viscous fluid permeated with suspended particles in a Brinkman porous medium. This stability effect is an agreement of the earlier work of Thirumurugan and Vasanthakumari [17]. Eq. (36) yields

$\frac{d R_{1}}{d S_{1}}=\left(\frac{G}{G-1}\right) \frac{B^{\prime}}{B}$

which is positive implying thereby the stabilizing effect of stable solute gradient on the double diffusive convection in Walters'B' elastico-viscous fluid permeated with suspended particles in a Brinkman porous medium. This stability effect is an agreement of the earlier work Sharma and Rana [4].

From the Eq.(36), we get

$\frac{\mathrm{dR}_{1}}{\mathrm{~dB}}=-\left(\frac{\mathrm{G}}{\mathrm{G}-1}\right)\left\{\left(\frac{(1+\mathrm{x})}{\mathrm{xPB}^{2}}\right)\left[1+(1+\mathrm{x}) \mathrm{D}_{\mathrm{A} 1}\right]-\frac{\mathrm{S}_{1} \mathrm{~B}^{\prime}}{\mathrm{B}^{2}}\right\}$

which is negative implying thereby the effect of suspended particles is destabilizing effect on the double diffusive convection Walters'B' elastic - viscous fluid in a Brinkman porous medium. This stability effect is an agreement of the earlier work of Scanlon and Segel [7] and Thirumurugan and Vasanthakumari [17]. It is evident from Eq. (36) that

$\frac{\mathrm{dR}_{1}}{\mathrm{dP}}=-\left(\frac{\mathrm{G}}{\mathrm{G}-1}\right)\left\{\left(\frac{(1+\mathrm{x})}{\mathrm{xBp}}\right)\left[1+(1+\mathrm{x}) \mathrm{D}_{\mathrm{A} 1}\right]\right\}$

From Eq. (41), we found that medium permeability has destabilizing effect on the double diffusive convection in Walters'B' elastico-viscous fluid permeated with suspended particles in a Brinkman porous medium, which is identical with the result as derived by Thirumurugan and Vasanthakumari [18].

The dispersion relation is analysed numerically and graphs have been represented by giving some numerical values for different parameters, to depict the stability characteristics. 


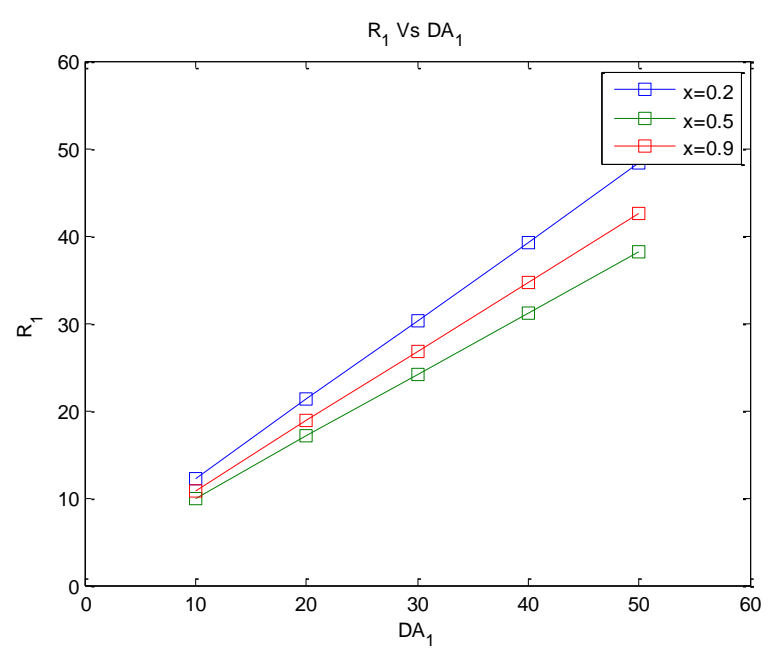

Figure 1. The variation of Rayleigh number $R_{1}$ with Darcy number $D_{A 1}$ for fixed values of $G=5, S_{1}=$ 4, $B=6, P=2, B^{\prime}=3$ and $x=0.2, x=0.5, x=0.9$

In Fig. 1, Rayleigh Number $R_{1}$ is plotted against Darcy number $D_{A 1}$ for $G=5, S_{1}=4, B=6, P=2$ and $B^{\prime}=3$ for fixed values of $x=0.2, x=0.5$ and $x=0.9$. As the value of Darcy number $D_{A 1}$ increases, the corresponding value of the Rayleigh number $R_{1}$ increases showing its stabilizing effect on the system.

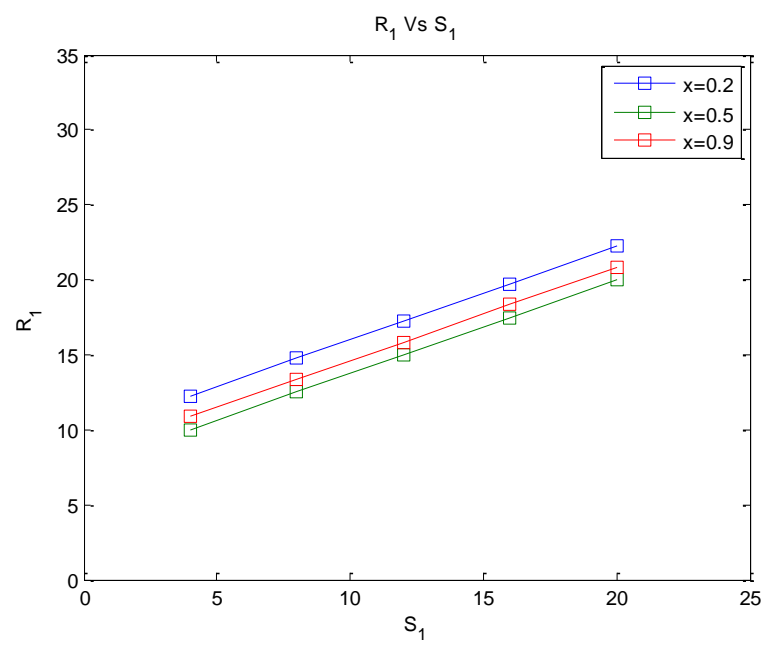

Figure 2. The variation of Rayleigh number $R_{1}$ with Stable solute gradient $S_{1}$ for fixed values of for $G=5$ and B $=6$,

$$
S_{1}=4, \mathrm{P}=2, B^{\prime}=3 \text { and } x=0.2, x=0.5, x=0.9
$$

In Fig. 2, Rayleigh number $R_{1}$ is plotted against Stable solute gradient $S_{1}$ for $G=5$ and $B=6, S_{1}=4, P$ $=2$ and $B=3$ for fixed wave numbers $x=0.2, x=$ 0.5 and $x=0.9$. The Rayleigh number $R_{1}$ increases with increases in stable solute gradient $S_{1}$ showing its stabilizing effect on the system.

In Fig. 3, Rayleigh Number $R_{1}$ is plotted against Suspended particles B for $G=5, S_{1}=4, B^{\prime}=3, \mathrm{P}=2$ and $D_{A 1}=10$ for fixed wave numbers $x=0.2, x=0.5, x=0.9$. The Rayleigh number $R_{1}$ decreases with the increases suspended particles $B$ showing its destabilizing effect on the system.

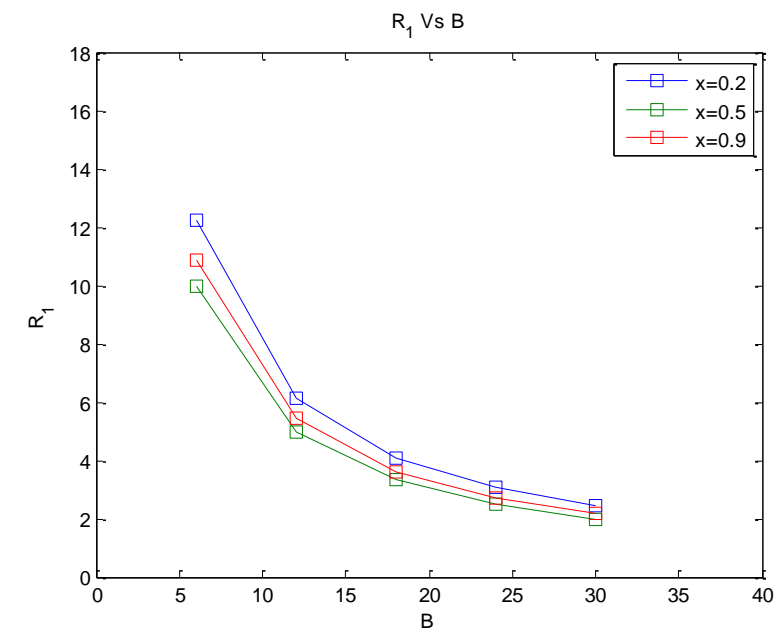

Figure 3. The variation of Rayleigh Number $R_{1}$ is plotted with Suspended particles $B$ for fixed values of $G=5, S_{1}=$ $4, B^{\prime}=3, P=2, D_{A 1}=10$ and $x=0.2, x=0.5, x=0.9$

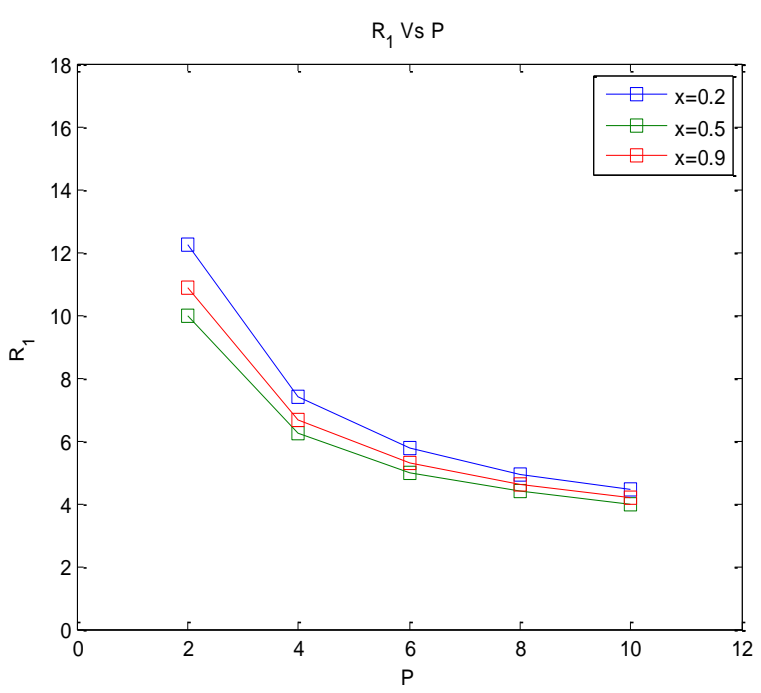

Figure 4. The variation of Rayleigh number $R_{1}$ with medium permeability $P$ for fixed values of $G=5, S_{1}=4, B=6$, $B^{\prime}=3$ and $D_{A 1}=10$ and $x=0.2, x=0.5, x=0.9$

In Fig. 4, Rayleigh number $R_{1}$ is plotted against medium permeability $P$ for $G=5, S_{1}=4, B=6 \quad B^{\prime}=3$ and $D_{A 1}=$ 10 for fixed wave numbers $x=0.2, x=0.5$ and $x=0.9$. The Rayleigh number $\mathrm{R}_{1}$ decreases with increases in medium permeability $P$ showing its destabilizing effect on the system.

\section{PRINCIPLE OF EXCHANGE OF STABILITIES AND OSCILLATORY MODES}

We have to examine the possibility of oscillatory modes, if any in compressible Walters' $\mathrm{B}^{\prime}$ viscoelastic fluid due to the presence of suspended particles, viscoelasticity, medium permeability and gravity field. Multiply Eq. (29) by $W^{*}$ the complex conjugate of $W$, integrating over the range of $z$ and according to Eq. (29), with the help of boundary conditions (Eq. (33)), we have 
$\left[1+\left(\frac{p_{l}}{\varepsilon}+\frac{M p_{l}}{\varepsilon\left(1+\tau_{1} \sigma\right)}+F\right) \sigma\right] I_{1}-D_{A} I_{2}-\frac{g a^{2} \alpha k p_{l}}{v \beta}$

$+\left(\frac{G}{G-1}\right)\left(\frac{1+\tau_{1} \sigma^{*}}{B+\tau_{1} \sigma^{*}}\right)\left(I_{3}+E p_{1} \sigma^{*} I_{4}\right) \frac{a^{2} \alpha^{\prime} g k^{\prime}}{v \beta^{\prime}}\left(\frac{1+\tau_{1} \sigma^{*}}{B+\tau_{1} \sigma^{*}}\right)$

$\left(I_{6}+E_{1} p_{1} \sigma^{*} I_{7}\right)=0$

where

$$
\begin{aligned}
& I_{1}=\int_{0}^{1}\left(|D W|^{2}+a^{2}|W|^{2}\right) d z \\
& I_{2}=\int_{0}^{1}\left(|D W|^{2}+2 a^{2}|W|^{2}+a^{4}|W|^{2}\right) d z \\
& I_{3}=\int_{0}^{1}\left(|D \Theta|^{2}+a^{2}|\Theta|^{2}\right) d z \\
& I_{4}=\int_{0}^{1}|\Theta|^{2} d z
\end{aligned}
$$

The integral part $I_{1}-I_{4}$ are all positive definite, putting $\sigma=i \sigma_{1}$ in Eq. (28), where $\sigma_{1}$ is real and equating the imaginary parts, we obtain

$$
\sigma_{i}\left[\begin{array}{c}
\left(\frac{p_{l}}{\varepsilon}+\frac{M p_{l}}{\varepsilon\left(1+\tau_{1}^{2} \sigma_{i}^{2}\right)}+F\right) I_{1}-\frac{g a^{2} d^{2} \alpha k p_{l}}{v \beta}\left(\frac{G}{G-1}\right) \\
\left\{\left(\frac{\tau_{1}(B-1)}{B^{2}+\tau_{1}^{2} \sigma_{i}^{2}}\right) I_{3}+\left(\frac{\tau_{1}(B-1)}{B^{2}+\tau_{1}^{2} \sigma_{i}^{2}}\right) E p_{1} I_{4}\right\} \\
-\frac{a^{2} \alpha^{\prime} g k^{\prime}}{v \beta^{\prime}}\left\{\left(\frac{\tau_{1}(B-1)}{B^{\prime 2}+\tau_{1}^{2} \sigma_{i}^{2}}\right) I_{5}+\frac{B^{\prime}+\tau_{1}^{2} \sigma_{i}^{2}}{B^{\prime 2}+\tau_{1}^{2} \sigma_{i}^{2}} E p_{1} I_{6}\right\}
\end{array}\right]=0
$$

Eq. (37) establishes that $\sigma_{i}=0$ or $\sigma_{i} \neq 0$ which shows that modes may be non - oscillatory or oscillatory modes employed due to the presence of viscosity, viscoelasticity, suspended particles and medium permeability which were non - existent in their absence.

\section{CONCLUSIONS}

The system has been investigated with the effect of suspended particles on double-diffusive convection of Walters'B' elastico-viscous fluid in the Brinkman porous medium. The dispersion relation, including the effects of suspended particles, Darcy number, medium permeability and viscoelasticity on the double-diffusive convection in Walters' $\mathrm{B}^{\prime}$ fluid in porous medium is derived mathematically. From the analysis, the main conclusions are as follows:

(i) For finding stationary convection, the compressible Walters'B' elastico-viscous fluid behaves like an ordinary Newtonian fluid as elastico-viscous parameter $F$ vanishes with $\sigma$.

(ii) The first derivative expressions $\frac{d R_{1}}{d D_{A 1}}, \frac{d R_{1}}{d S_{1}}, \frac{d R_{1}}{d B}$, and $\frac{d R_{1}}{d P}$ have been examined analytically and it has been found that the Darcy number and stable solute gradient have stabilizing effect whereas the suspended particles and medium permeability have a destabilizing effect on the system.

(iii) The effects of Darcy Number, stable solute gradient and medium permeability on double-diffusive convection in Walters'B' elastico - viscous fluid permeated with Suspended particles, in a Brinkman porous medium is also represented graphically as in Figs. 1, 2, 3 and 4 respectively, which are good agreement with the analytical results.

(iv) The oscillatory modes are employed to examine the nature of stability existent due to the presence of viscoelasticity, suspended particles, gravity field and medium permeability, which were non - existent in their absence.

\section{ACKNOWLEDGMENT}

The authors are grateful to the reviewers and editors for their useful comments and valuable suggestions.

\section{REFERENCES}

[1] Chandrasekhar. S., Hydrodynamics and Hydromagnetics Stability, New York: Dover Publication, 1981.

[2] Veronins, G., "On finite amplitude instability in thermohaline convection," J, Marine research, vol. 23 pp. $1-17,1965$.

[3] Stommel, H. and Fedorov, K. N., "Small scale structure in temperature and salinity near timor and mindano," Tellus, vol. 19, pp. 306-325, 1967. DOI: $\underline{10.1111 / j .2153-3490.1967 . t b 01484 . x}$.

[4] Sharma, V and Rana, G. C., "Themosolutal instability of a Walters' (Model B') elastic-viscous rotating fluid in the presence of magnetic field and variable gravity field in porous medium," Proc. Nat. Acad. Sci, India, 73, pp. 93-111, 2003.

[5] Brakke, M. K., "Zone electrophoresis of dyes, proteins and viruses in density-gradient columns of sucrose solutions solutions," Arch. Biochem. Biophysics, vol. 55, pp. 175-190, 1955. DOI: 10.1016/00039861(55)90556-7.

[6] Nason, P., Schumaker, V. Halsall, B. and Schweds, J., "Formation of streaming connective disturbance which may occur at one gravity during preparation of samples for zone centrifugation," Biopolymers, vol. 7, pp. 241-249, 1969.

DOI:

10.1002/bip.1969.360070210.

[7] Scanlon, J. W. and Segel, L. A. "Some effect of suspended particles on the onset of Bénard convection," Physics Fluids, vol. 16, pp. 1573 -1578, 1973. DOI: $10.1063 / 1.1694182$.

[8] Palaniswamy, V. I. and Purushotham, C. M., "Stability of a shear flow of stratified fluids with fine dust," Phys. Fluids, vol. 24, pp. 1224-1229, 1981. DOI: $10.1063 / 1.863523$.

[9] Spiegel, E. A. and Veronis, G., "On the Boussinesq approximation for a compressible fluid," Astrophys. J., vol. 131, pp. 442, 1960. DOI: 10.1086/146849.

[10] Bhatia, P. K. and Steiner, J. M., "Convective instability in a rotating viscoelastic fluid layer," $Z$. Angew. Math. Mech., vol. 52, pp. 321-327, 1972. DOI: $10.1002 /$ zamm.19720520601.

[11] Sharma, R.C., "Effect of rotation on thermal instability of a viscoelastic fluid layer," Actaphysics Hung., vol.40, pp. 11-17, 1976. DOI: 10.1007/BF03157148.

[12] Walters, K., "Non-Newtonian effect in some elasticviscous liquids whose behavior at some rates of shear 
in characterized by a general linear equations of state," J. Mech. Appl. Math, 15, pp. 63-75, 1962.

[13] Chandra, K., "Instability of fluids heated from below," Proceeding of Royal Society London, A164, pp. 231242, 1938. DOI: $10.1098 /$ rspa.1938.0015.

[14] Lapwood. E. R., "Convection of a fluid in porous medium," Mathematical Proceedings of the Cambridge Philosophical Society, vol. 44, pp. 508519, 1948. DOI: $\underline{10.1017 / S 030500410002452 X}$.

[15] Wooding. R. A., "Rayleigh instability of thermal boundary layer in flow through a porous medium," Journal of fluid mechanics, vol. 9, pp. 183-192, 1960. DOI: 10.1017/S0022112060001031.

[16] Parul Aggarwal and Urvasi Gupta., "Double-diffusive of compressible rotating walters'B fluid with hall currents saturating a porous medium," International Journal of Engineering, Science and technology, vol. 4, no. 2, pp. 137-151, 2012. DOI: 10.4314/ijest.v4i2.10.

[17] Thirumurugan, K. and Vasanthakumari, R., "Thermal convection in Walters'B' viscoelastic fluid in DarcyBrinkman porous medium with effect of dusty particles," Int. Journal of Innov. Research in Sci. Eng and Tech, vol. 2, no. 8, pp. 3964-3971, 2013. http://www.ijirset.com/upload/august/35A_THERMA L.pdf.

[18] Thirumurugan, K. and Vasanthakumari, R., "Hydromagnetics instability of Non-Newtonian Walters'B' viscoelastic rotating fluid in porous medium," World Journal of Engineering, vol. 11, no. 4, pp. 365-372, 2014. DOI: 10.1260/17085284.11.4.365.

\section{NOMENCLATURE}

$\mathrm{C}_{\mathrm{f}}$

$\mathrm{C}_{\mathrm{pt}}$

$\mathrm{C}_{\mathrm{s}}$

$\mathrm{C}_{\mathrm{f}}^{\prime}, \mathrm{C}_{\mathrm{pt}}{ }^{\prime}$

$\mathrm{p}$

$\mathrm{N}$

$\mathrm{K}$

$\mathrm{K}$

$\mathrm{K}_{\mathrm{T}}$

$\mathrm{k}_{1}$

$\mathrm{t}$

$\mathrm{T}$

H

$\rho$

$\rho_{\mathrm{s}}$

$\rho_{0}$

v

$\eta$

$\mu_{\mathrm{e}}$

$\delta \mathrm{p}$

$\delta \rho$

$\varepsilon$

$\beta$

$\theta$

$\alpha$

$v^{\prime}$

$\alpha^{\prime}$

$\beta^{\prime}$
Fluid velocity

Heat capacity of fluid

Heat capacity of particles

Heat capacity of solid

Heat capacity analogous to solute

Pressure

Suspended particles number density Thermal diffusivity

Solute diffusivity

Thermal conductivity

Medium permeability

Time coordinate

Temperature

Acceleration due to gravity

Magnetic field

Fluid density

Density of solid

Reference density

Kinematic viscosity

Particle radius

Magnetic permeability

Perturbation in pressure

Perturbation in density

Medium porosity

Temperature gradient

Perturbation at temperature $\mathrm{T}$

Thermal coefficients of expansion

Kinematic viscoelasticity

Solvent coefficients of expansion

Solute gradient 